\title{
Magnetic Helicity and Filaments
}

\author{
M. A. Berger \\ Mathematics, University College London, London WC1E 6BT, U.K.
}

\begin{abstract}
Some of the most dramatic images of prominences show helical structure. Helical structure, as well as other structural features such as twist, shear, and linking, can be quantified using helicity integrals. This paper reviews how the calculation of helicity may be applied to prominence models. Recent observations indicate that the sign of helicity in an active region depends on which hemisphere the region is in. The source of this asymmetry is an important problem in solar physics. The total helicity of each hemisphere obeys a Poynting-like theorem which describes how helicity is transferred across the photosphere and the equator. Estimating this helicity transfer may help us in understanding the helicity balance of the sun.
\end{abstract}

\section{Introduction}

Some four decades ago, the problems of geomagnetism (Elsasser 1956) and solar magnetism (Woltjer 1958) motivated the discovery of magnetic helicity as a conserved quantity in MHD. Unfortunately, magnetic helicity has often been perceived to be difficult to calculate. Section 2 will try to show that the calculation of helicity for many coronal geometries is not difficult: in many cases the helicity reduces to a sum of terms which only involve measures of twist, shear, and flux. Helicity has several nice properties which make its calculation worthwhile: it is conserved in ideal MHD and approximately conserved during reconnection, it quantifies structural features of the field such as twist, shear, kinks, and linking, and it can be computed in any geometry. Section 3 gives an example calculation, showing where the helicity resides in a simple model of a twisted prominence.

Helicity allows us to compare models of fields in different geometries. Thus, a model of a sheared arcade might use its own special parameter to measure the net shear, while models of twisted flux tubes may have their own special parameters to measure net twist. The situation becomes even worse if different authors working on the same basic geometry define their parameters differently. Helicity integrals provide a single universal parameter to measure structural features like twist and shear, thus avoiding the use of parameters specific to one model. Also, if a magnetic system is described in terms of interacting parts (e.g., the solar field as subphotospheric + coronal, or a prominence system as filament + barbs + overlying arcade), then one can describe changes in structure in terms of how helicity is transferred from one part to another.

Helicity is approximately conserved in high magnetic Reynolds number plas- 


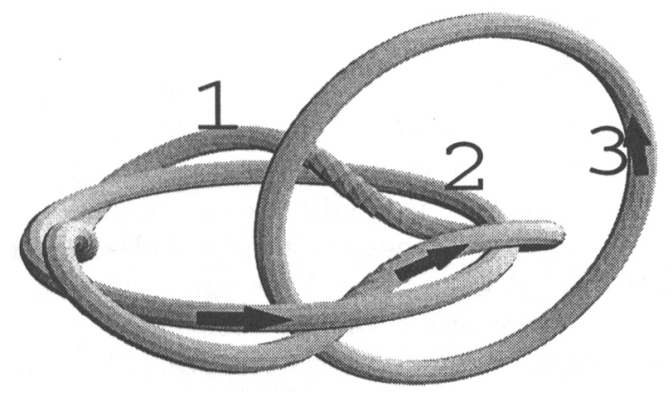

Figure 1. A set of three linked tubes with linking numbers $L_{12}=3$, $L_{13}=L_{23}=-1$.

mas (Berger 1984, Freedman and Berger 1993). Approximate helicity conservation provides a valuable constraint on the evolution of a reconnecting field. Of course, one can do physical calculations without worrying about conserved quantities; just let the evolution equations (e.g., Navier-Stokes and magnetic induction) do their work. One might think of calculating orbits of planets about the sun. This can be done numerically without invoking energy and angular momentum; however, these two quantities are essential for understanding the orbits (as well as for finding analytic solutions). Similarly, consideration of where magnetic helicity and magnetic energy come and go can provide shortcuts to solutions of MHD problems as well as checks on numerical calculations. In a confined volume, widespread reconnection may reduce the field to its minimum energy state, given the amount of helicity. This state is a linear force-free field (Heyvaerts and Priest 1984, Dixon et al. 1989). Insufficient reconnection will leave the field in a higher energy state, e.g., a nonlinear force-feee field.

The build-up of helicity in active regions can influence the structure of prominences both before and after eruption; helicity build-up may even be the cause of eruptions (Low 1994, Rust 1994). Furthermore, recent observations (Seehafer 1990, Martin et al. 1992, Pevtsov et al. 1994, Rust and Kumar $1994,1996)$ have suggested that the sign of helicity in active regions is primarily negative in the Northern hemisphere and positive in the Southern hemisphere. The source of this asymmetry may arise below the photosphere in the solar dynamo (van Ballegooijen and Martens 1990, Rust 1996, Ruzmaikin 1996, Seehafer 1996). Section 4 shows how observations of differential rotation of open flux may be useful in understanding where the helicity comes from. 


\section{A Primer on Calculating Magnetic Helicity}

In 1833 Carl Gauss introduced the idea of linking number. More recent discoveries by Faraday and others had revealed close relations between electric currents and magnetic fields; Gauss became intrigued by the mathematical problems raised by inductance (where the net electric force around a circuit equals the rate of change of the magnetic field linked by the circuit). The linking number $L$ (see Figure 1) is a topological number describing two closed curves. Each curve is given a direction; reversing one of the directions multiplies $L$ by -1 . No matter how the curves are distorted, $L$ does not change unless the curves pass through each other. Gauss showed that $L$ could be expressed as a certain line integral along the curves (see, for example, Moffatt 1969). While this integral is rarely used in practice to calculate $L$, it is useful in proving theorems about $L$, and provides a bridge between the intuitive world of geometrical pictures such as Figure 1, and the analytic world of integrals and vector calculus.

The linking of closed curves may at first seem irrelevant to the geometry of coronal loops. Note, however, that curves 1 and 2 in Figure 1 twist about each other - their linking number $L_{12}$ measures how much twisting. The twist of two filaments about each other can sometimes be seen in erupting prominence images
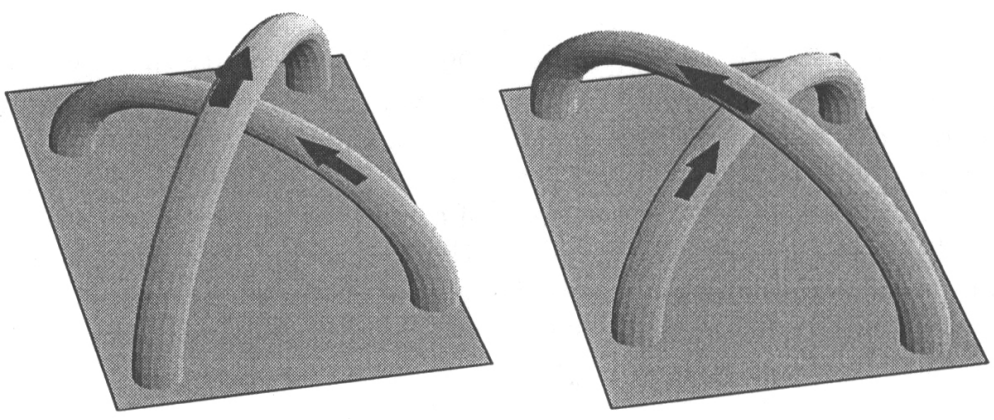

Figure 2. A crossing can be either positive (left) or negative (right).

(House and Berger 1987, Vršnak et al. 1991). Furthermore, in any diagram or image involving curves or filaments there will be special points where two curves are seen to cross over each other. For example, the lines of force in an overlying arcade may cross over the lines in the prominence filament. The topology of both closed curves and open curves (i.e., curves with endpoints on a boundary such as the photosphere) can be examined using the notion of crossings.

In fact, crossings provide a nice way of calculating linking numbers while avoiding doing line integrals. Each crossing of the curves in Figure 1 has a sign +1 or -1 (see Figure 2). The linking number of closed curves is precisely half 
the sum of signed crossings. This sum does not vary with either viewing angle of the curves or deformation of the curves. You do not need to carry Figure 2 with you whenever you feel the need to calculate $L$ - there are some simple rules for finding the sign. First, the blades in ordinary right-handed scissors orient themselves to make a positive crossing; if you wish to purchase good quality left-handed scissors, make sure the crossing is negative. Alternatively, imagine two one-way roads which cross at a bridge. Let traffic flow in the direction of the arrows. For a positive crossing, the traffic on the upper road goes to the right as seen by drivers approaching the bridge on the lower road. A third method consists of the right hand rule. This is perhaps easiest looking at the complete closed curves as in Figure 1. Curl the fingers of your right hand along one of the curves (say curve 2) in the direction of its arrow. Now look at one of the places where curve 2 crosses over curve 3 . If the crossing is positive, then the thumb will point in the direction of curve 3 .

The concept of the linking of curves extends to the linking and twisting of magnetic field lines (Moffatt 1969). The magnetic helicity, very crudely, is the sum over linking numbers over all pairs of field lines. Field lines, of course, are difficult to work with: they are infinitesimally thin and there are infinitely many of them. It is far better to think of the net linking of magnetic flux. The magnetic helicity integral is a double volume integral similar in form to the Gauss integral. As applied to Figure 1, it gives a total helicity of

$$
H=T_{1} \Phi_{1}^{2}+T_{2} \Phi_{2}^{2}+T_{3} \Phi_{3}^{2}+2 L_{12} \Phi_{1} \Phi_{2}+2 L_{13} \Phi_{1} \Phi_{3}+2 L_{23} \Phi_{2} \Phi_{3} .
$$

Here $\Phi_{1}$ is the net flux of tube 1 and $T_{1}$ refers to the net linking of field lines within tube 1. For a uniformly twisted circular tube, $T$ measures the net number of turns (through $2 \pi$ ) that a field line makes about the central axis. We can call $T_{1} \Phi_{1}^{2}$ the self helicity of tube 1 and $2 L_{12} \Phi_{1} \Phi_{2}$ the mutual helicity between tubes 1 and 2 , in analogy with self and mutual induction.

So far we have discussed closed flux tubes. But coronal fields are rooted at the photosphere and do not form closed curves. In general, helicity integrals (like electrical voltage) require a ground state which sets the zero. The potential field $(\nabla \times \mathbf{B}=0)$ fits this role well: given the distribution of flu $\mathbf{x}$ at the boundary $(\mathbf{B} \cdot \hat{n})$, the potential field minimises the energy. With the helicity of the potential field set to 0, the helicity is always uniquely specified (Berger and Field 1984, Jensen and Chu 1984, Finn and Antonsen 1985).

Figures 3 and 4 show the magnetic helicity of various configurations with a plane boundary. First, consider a single twisted tube, Figure 3 (left). In general, the interior of a flux tube may not be uniformly twisted. In this case the helicity provides a measure of overall twist. Suppose the tube consists of a set of nested flux surfaces. Let $\phi$ be the axial flux within a particular surface. Thus, the central axis has $\phi=0$, while the outermost surface has $\phi=\Phi$, the total axial flux. Also let $T(\phi)$ equal the twist (number of turns about the axis) at the flux surface labelled by $\phi$. Then

$$
H=2 \int_{0}^{\Phi} T(\phi) \phi d \phi
$$

Dividing $H$ by $\Phi^{2}$ gives a dimensionless twist parameter. A highly twisted tube may begin to kink (Hood and Priest 1979, Velli et al. 1997). The amount of 

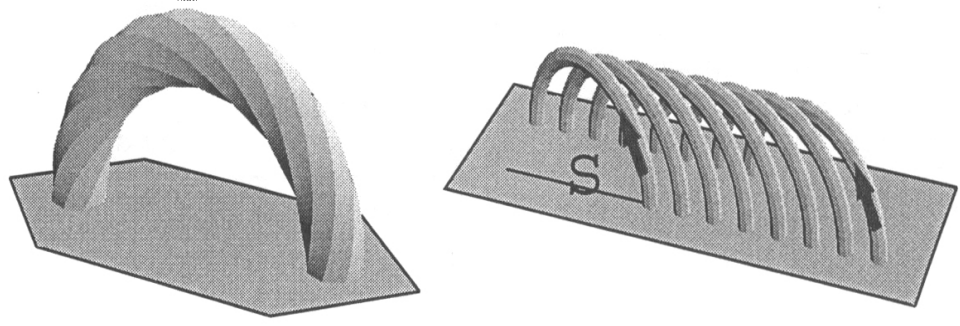

Figure 3. A twisted flux tube and a sheared arcade.
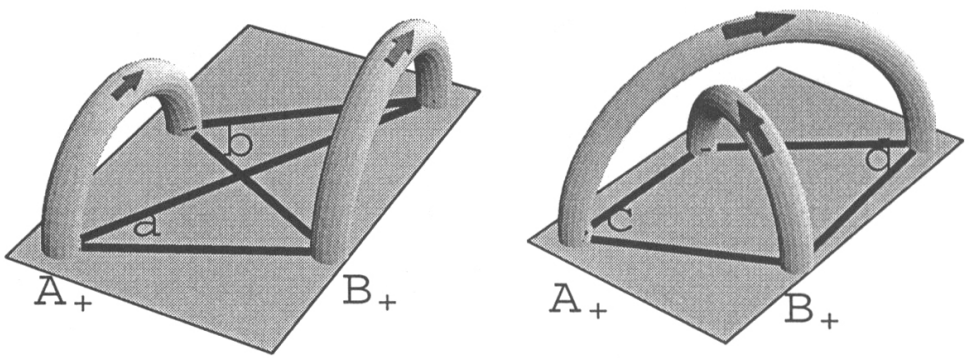

Figure 4. Mutual helicity. For the tubes on the left, the mutual helicity is $H_{A B}=(a-b) \Phi_{A} \Phi_{B} / \pi$. On the right, $H_{A B}=(c+d) \Phi_{A} \Phi_{B} / \pi$.

kinking of the central axis can be quantified by the writhing number (Moffatt and Ricca 1992). In general the self helicity of the tube can be described as equalling the sum of the writhing number due to the shape of the axis, plus additional twist of field lines about the axis.

Next consider the arcade in Figure 3. This arcade has infinite extent, so we must calculate the helicity per unit length, $\tilde{H}$. The arcade has flux per unit length, $\tilde{\Phi}$, and has been sheared by the length, $S$. Then

$$
\tilde{H}=S \tilde{\Phi}^{2} .
$$


Figure 4 shows two examples of pairs of coronal arches. We assume that the footpoints of the arches are small compared to the distance between the footpoints. Let $\operatorname{arch} A$ have positive footpoint $A_{+}$and negative footpoint $A_{-}$, and similarly for $\operatorname{arch} B$. To calculate the mutual helicity between $A$ and $B$, find the angles $\angle B_{+} A_{+} B_{-}$and $\angle B_{+} A_{-} B_{-}$. The mutual helicity is

$$
H_{A B}=\left(\angle B_{+} A_{-} B_{-}-\angle B_{+} A_{+} B_{-}\right) \Phi_{A} \Phi_{B} / \pi .
$$

The plane boundary can be easily replaced with a spherical boundary; simply replace ordinary angles in Figure 4 by a spherical angle. (Consider 3 points $P, Q, R$ on a sphere. The spherical angle $\angle P Q R$ at vertex $Q$ can be found by rotating the coordinates so that $Q$ is at the North pole; then $\angle P Q R$ equals the difference in longitude between $P$ and $R$.)

\section{Example: A Sheared Arcade Reconnecting to Form a Twisted Prominence}

Many authors have suggested that twisted flux tubes can support prominence material (Hood and Priest 1979, Pneuman 1983, van Ballegooijen and Martens 1989, Priest, Hood and Anzer 1989, Ricca 1997). Here we consider a simple model where an initially sheared arcade partially reconnects (Figure 5). The part that does not reconnect can be called the overlying arcade (region 1). The reconnecting flux transforms into a twisted tube and an underlying arcade (regions 2 and 3 ). The fields are assumed invariant in the $x$ direction. We choose the initial flux at the photosphere, $z=0$, to be linear,

$$
B_{z}=-B_{0} y / a, \quad|y| \leq a
$$

with no flux entering the corona outside $|y|=a$. The shear profile is also linear: footpoint displacements occur in the $x$ direction with magnitude

$$
\Delta x(y)=-\frac{s y}{2 a} \text {. }
$$

Thus, for example, a field line starting at $(x, y, z)=\left(x_{0},-y_{0}, 0\right)$ re-enters the photosphere at $\left(x_{0}-s y_{0} / a, y_{0}, 0\right)$.

The helicity per unit length of this initial configuration can be computed using equation (3):

$$
\tilde{H}=\int_{-a}^{a} \int_{y}^{a} B_{z}(y) B_{z}\left(y^{\prime}\right)\left(\Delta x\left(y^{\prime}\right)-\Delta x(y)\right) d y^{\prime} d y .
$$

The result is $\tilde{H}=2 B_{0}^{2} s a^{2} / 15$.

We suppose that only the photospheric flux within $|y|=\epsilon a$ reconnects, where $\epsilon$ is some number between 0 and 1 . In the final configuration, the flux inside $|y|=\epsilon a$ belongs to the underlying arcade, while the outer flux goes into the overlying arcade. By an integration similar to equation (7), the self helicity (per unit length) of the overlying arcade is

$$
\tilde{H}_{11}=\left(1-5 \epsilon^{3} / 2+3 \epsilon^{5} / 2\right) \tilde{H} .
$$




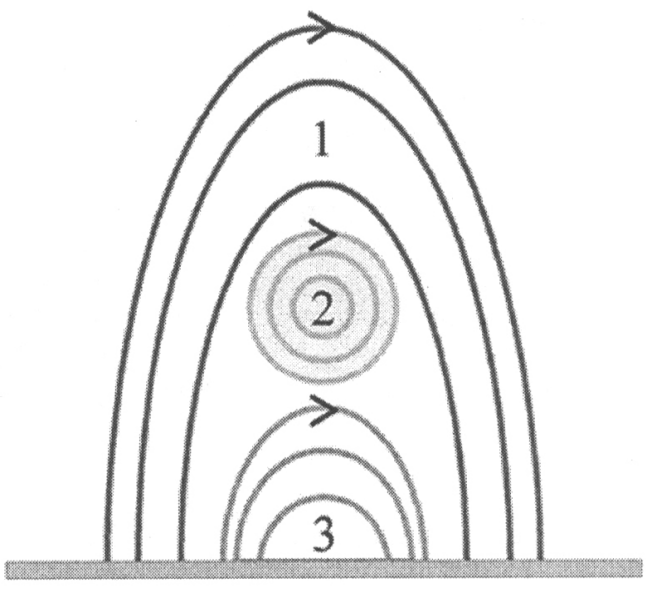

Figure 5. A twisted prominence model.

Meanwhile the underlying arcade no longer contributes any helicity. Its self helicity $\tilde{H}_{33}=0$ by the assumption of zero final shear. Its mutual helicities with the overlying arcade and the central flux tube also vanish.

The central tube (region 2) has self helicity

$$
\tilde{H}_{22}=\epsilon^{5} \tilde{H}
$$

this can be calculated using equation (2). Also the tube has flux $\Phi_{2}=\epsilon^{3} B_{0} s a / 3$. The mean twist parameter (per unit length again) is $\tilde{H}_{22} / \Phi_{2}^{2}=6 /(5 \epsilon s)$.

Finally, the azimuthal ( $y$ direction) flux in the overlying arcade is $\tilde{\Phi}_{1}=$ $B_{0}\left(1-\epsilon^{2}\right) / 2$. This flux links the axial tube flux; thus the mutual helicity is

$$
\tilde{H}_{12}=2 \tilde{\Phi}_{1} \Phi_{2}=5\left(\epsilon^{3}-\epsilon^{5}\right) / 2 .
$$

Note that

$$
\tilde{H}=\tilde{H}_{11}+\tilde{H}_{12}+\tilde{H}_{22} .
$$

For example, if $\epsilon=3 / 4$, then

$$
\begin{aligned}
& \tilde{H}_{11}=0.30 \tilde{H}, \\
& \tilde{H}_{12}=0.46 \tilde{H}, \\
& \tilde{H}_{22}=0.24 \tilde{H} .
\end{aligned}
$$

Thus the helicity is shared between shear $\tilde{H}_{11}$, linking $\tilde{H}_{12}$, and twist $\tilde{H}_{22}$. Any additional reconnection, for example at the initiation of a mass ejection, could change these ratios. 


\section{Helicity Balance in the Northern Hemisphere}

Ruzmaikin (1996) and Seehafer (1996) explore the possibility that the $\alpha$ effect in dynamo theory causes the observed asymmetry in active region helicity in the two hemispheres of the sun. The $\alpha$ effect does not create any net helicity; instead it creates opposite signs of helicity in the mean field and the fluctuating field. Classically the mean field in the North should get positive helicity. If this is indeed happening below the photosphere, then the negative helicity seen in active regions must come from the fluctuating field. This scenario may be seen as a consequence of helicity conservation during reconnection. If two flux tubes reconnect deep below the photosphere, they will change their mutual helicity with an equal and opposite change in self helicity (Song and Lysak 1989, Wright and Berger 1989). The self helicity is stored in twist internal to the flux tubes, which in mean field theory would appear in the fluctuating part of the field.

On the other hand, the net helicity of the North may be negative. Let $H_{N}$ be the total magnetic helicity of all magnetic fields in the solar interior north of the equator. Ignoring the small resistive dissipation term, the time derivative of $H_{N}$ can be shown to a surface integral (Berger 1984):

$$
\begin{aligned}
\frac{d H_{N}}{d t} & =\frac{1}{2 \pi} \iint_{\text {surface }} B_{r}(\mathbf{a}) B_{r}\left(\mathbf{a}^{\prime}\right) \frac{d \varphi\left(\mathbf{a}, \mathbf{a}^{\prime}\right)}{d t} d^{2} a d^{2} a^{\prime} \\
& -\frac{1}{2 \pi} \iint_{\text {equator }} B_{z}(\mathbf{x}) B_{z}\left(\mathbf{x}^{\prime}\right) \frac{d \theta\left(\mathbf{x}, \mathbf{x}^{\prime}\right)}{d t} d^{2} x d^{2} x^{\prime}
\end{aligned}
$$

where $\mathbf{a}, \mathbf{a}^{\prime}$ are points on the surface and $d \varphi\left(\mathbf{a}, \mathbf{a}^{\prime}\right) / d t$ is the angular velocity of the field line at $\mathbf{a}$ about the field line at $\mathbf{a}^{\prime}$ due to fluid motions. Similarly, $\mathbf{x}$ and $\mathbf{x}^{\prime}$ label points on the equatorial plane below the surface; these rotate about each other with angular velocity $d \theta\left(\mathbf{x}, \mathbf{x}^{\prime}\right) / d t$. This Poynting-like equation describes transfer of helicity into and out of the Northern hemisphere.

Part of the transfer comes from differential rotation (Rust 1996). Open field lines in Northern coronal holes rotate, sending negative helicity into the Parker spiral. Conservation of helicity implies that an equal and opposite positive helicity goes downwards into the sun (this contributes to the first term in equation 15). But the net open flux leaving the Northern hemisphere must equal the amount of flux entering through the equatorial plane (mostly at the base of the convection zone). This flux also rotates, sending negative helicity upwards (second term in equation 15), and positive helicity downwards. If rotation is faster at the equator, then negative helicity will build up in the Northern hemisphere. This helicity may then find its way into active regions, eventually to be released in coronal mass ejections. Of course, during the solar cycle, toroidal flux crosses the equator cancelling out some of the helicity buildup. Both observations of the distribution of open flux and theoretical studies of the solar dynamo will be needed to understand the helicity balance of the sun.

\section{References}

Berger, M.A. and Field, G.B. 1984, J. Fluid Mechanics, 147, 133

Berger, M.A. 1984, Geophys. Astrophys. Fluid Dynamics, 34, 265

Dixon, A., Berger, M.A., Browning, P. and Priest, E.R. 1989, 225, 156 
Elsasser, W.M. 1956, Rev. Modern Phys., 28, 135

Finn, J.H. and Antonsen, T.M. 1985, Comments Plasma Phys. Contr. Fusion, 9,111

Freedman, M. and Berger, M.A. 1993, Geophys. Astrophys. Fluid Dynamics, 73,91

Heyvaerts, J. and Priest, E.R. 1984, A\&A, 137, 63

Hood, A. and Priest, E.R. 1979, Solar Phys., 64, 303

House, L. and Berger M.A. 1987, ApJ, 323, 406

Jensen, T. and Chu, M.S. 1984, J. Plasma Phys., 25, 459

Low, B.C. 1994, Phys. Plasmas, 1, 1684

Martin, S.F. et al. 1992, in The Solar Cycle, (ed.) K.L. Harvey, ASP Conf. Ser. Vol. 27, San Francisco, p. 53

Moffatt, H.K. 1969, J. Fluid Mechanics, 35, 117

Moffatt, H.K. and Ricca, R.L. 1992, Proc. Royal Soc. Lon. A, 439, 411

Pevtsov, A.A., Canfield, R.C. and Metcalf, T.R. 1994, ApJ, 440, L109

Priest, E.R., Hood, A.W. and Anzer U. 1989, ApJ, 344, 1010

Pneuman, G.W. 1983, Solar Phys., 88, 219

Ricca, R.L. 1997, Solar Phys., 172, 241

Rust, D. 1994, Geophys. Res. Lett., 21, 241

Rust, D. 1997 in Coronal Mass Ejections, (eds.) N. Crooker, J. Joselyn, and J. Feynman, AGU G.M. 99, Washington, D.C., p. 119

Rust, D. and Kumar, A. 1994, Solar Phys., 155, 69

Rust, D. and Kumar, A. 1996, ApJ, 464, L199

Ruzmaikin 1996 Geophys. Res. Lett., 23, 2649

Seehafer N. 1990, Solar Phys., 125, 219

Seehafer N. 1996, Phys. Rev. E, 53, 1283

Song, Y. and Lysak, R.L. 1989, J. Geophys. Res., 94, 5273

van Ballegooijen, A.A. and Martens, P.C.H. 1990, ApJ, 361, 283

Velli, M., Lionello, R. and Einaidi, G. 1997, Solar Phys., 172, 257

Vršnak, B., Ruždjak, V. and Rompolt, B. 1991, Solar Phys., 136, 151

Woltjer, L. 1958, Proc. Natl. Acad. Sci. USA, 44, 489

Wright, A. and Berger, M.A. 1989, J. Geophys. Res., 94, 1295 